\title{
L'observation visuelle. Regards croisés d'un agronome et d'un géographe
}

\author{
Jean-Pierre Deffontaines ${ }^{\mathrm{a}, \mathrm{t}}$, Patrick Caron ${ }^{\mathrm{b}}$ \\ a Agronome \\ b Géographe, CIRAD, 73 rue J.-F. Breton, TA 60/15, 34398 Montpellier cedex 5, France
}

C'est un paradoxe de la vision d'être à la fois source d'échange et regard individuel et unique. Il y a en effet une infinité de regards sur un même objet, liée à l'histoire de chaque regardant: «La vision est la rencontre, comme à un carrefour, de tous les aspects de l'être ${ }^{1}$. » L'observation mobilise, d'une manière particulière, la vision. Elle est un regard associé à une intention. Sans doute est-ce à cause de ce paradoxe que l'observation visuelle a eu du mal à se faire reconnaître comme moyen d'investigation scientifique.

Au milieu du XX $X^{\mathrm{e}}$ siècle, cependant, à l'instar de l'expérience, l'observation visuelle acquiert un statut scientifique. On s'éloigne d'une exclusive accordée au modèle expérimental de Claude Bernard. Dans le champ de l'agronomie, en particulier, la référence à la définition proposée par $\mathrm{S}$. Hénin, celle d' $\mathrm{d}^{\prime}$ une écologie appliquée à la production de peuplements végétaux et à l'aménagement du territoire », revient à admettre que tout ne se mesure pas, ne s'expérimente pas, et que les approches qualitatives et globales ont leur place dans l'acquisition des connaissances, dans l'élaboration de diagnostics, voire dans la modélisation des processus en cause. Dans l'introduction de l'ouvrage Le Profil cultural ${ }^{2}$, on note les propos suivants : «En définitive nous avons été conduits à abandonner l'attitude trop stricte du physicien qui conduit

\section{Auteur correspondant : P. Caron, patrick.caron@cirad.fr}

Après la disparition de Jean-Pierre Deffontaines au cours de la rédaction, son co-auteur a souhaité finaliser ce texte en s'aidant de leurs notes respectives. Dans l'état, ce texte a pour simple ambition d'ouvrir des pistes qui peuvent interpeller des chercheurs de différentes disciplines.

1 Merleau-Ponty M., 1964, L'CEil et l'esprit, Paris, Gallimard.

${ }^{2}$ Hénin, S., Gras, R., Monnier, G., 1969 (2 éd. entièrement refondue). Le Profil cultural : l'état physique du sol et ses conséquences, Paris, Masson. à chercher immédiatement à exprimer les faits uniquement en termes de grandeur mesurable. Nous cherchons à adopter une attitude plus réaliste en commençant l'analyse du milieu à partir de concepts globaux moins précis mais plus significatifs. C'est l'attitude du médecin : la méthode est qualitative. Nous pensons qu'une méthode qualitative peut être rendue systématique [...]. Parmi les symptômes, il en est de visuels qui peuvent être appréciés directement sur le terrain et qui sont si typiques qu'ils entraînent immédiatement une conclusion ; $\mathrm{d}$ 'autres sont moins évidents et nécessitent une confirmation que l'on demande aux méthodes analytiques. " Par cette démarche, ces agronomes affirment l'importance de l'observation visuelle et lui reconnaissent un statut scientifique. On prend ainsi conscience que la nature ne se réduit pas à des phénomènes qui peuvent être expérimentés. Les limites de l'expérience rencontrées par l'écologie et les sciences humaines rapprochent les deux domaines scientifiques. "C'est sans doute l'un des progrès épistémologiques essentiels de la seconde moitié du $\mathrm{XX}$ e siècle $[\ldots]^{3}$. »

Face à ces brèves réflexions sur l'observation visuelle, nous faisons le constat, en nous référant à nos expériences professionnelles respectives, de l'importance du rôle de l'observation dans nos pratiques de recherche, en agronomie comme en géographie, et, simultanément, de la rareté des réflexions sur ce moyen majeur de travail. Ce constat est à l'origine du projet de formuler et de préciser la place et les conditions de l'observation - si ce n'est dans nos disciplines, tout du moins dans nos recherches.

\footnotetext{
${ }^{3}$ Legay, J.-M., Deffontaines, J.-P., 1992. Complexité, observation et expérience, in Jollivet, M. (Ed.), Sciences de la nature, sciences de la société : les passeurs de frontières, Paris, CNRS Éditions, $477-485$ (cit. p. 479-480).
} 
Après avoir pris conscience que «l'observation ne va pas de $\operatorname{soi}^{4} »$, nous avons mis en avant quelques questions :

- Comment observer? Comment identifier des traces et des signes visuels pertinents? Quel sens leur donner et quel domaine de validité ont-ils? Y a-t-il des critères de scientificité de l'observation visuelle? Peut-on élaborer un schéma d'observation?

- Y a-t-il des conditions et comment se mettre en situation d'observation?

- Y a-t-il une aptitude, une curiosité intellectuelle ou un instinct à observer? Comment faire advenir des questions telles que : comment cela marche ou pourquoi les choses sont-elles comme cela?

- Y a-t-il des processus d'apprentissage? L'observation s'enseigne-t-elle ou est-elle de l'ordre de la contagion?

Le champ couvert par ces interrogations est vaste, aussi avons-nous cherché à le réduire en définissant quelques orientations de travail qui structurent notre propos. Nous nous concentrons sur l'observation proprement dite, son processus et ses pratiques. Nous n'abordons pas la communication, l'apprentissage et la formation à l'observation. Par ailleurs, nous nous référons à l'expérience acquise sur l'observation du paysage. Celleci est destinée à comprendre les pratiques mises en œuvre dans le territoire par les éleveurs et les agriculteurs dans l'exercice de leur activité de production. C'est l'objet de la partie intitulée "Deux situations d'observation ». Il a fallu construire nos itinéraires respectifs d'observation et les analyser a posteriori dans la confrontation et la comparaison. Le but est de faire ressortir quelques traits généraux sur les pratiques d'observation dans nos métiers de recherche en agronomie et en géographie. Notre hypothèse est que, si chaque chercheur développe une trajectoire évolutive d'observation qui lui est propre, il existe dans ces pratiques des traits communs, des constantes, susceptibles de participer à des énoncés enseignables sur l'observation. Cette partie est appelée «Une mise en correspondance des pratiques d'observation ». À partir de cette analyse, la dernière partie propose une réflexion sur le rôle de l'observation visuelle dans les processus de production de connaissances.

\section{Deux situations d'observation}

Il nous semble éclairant de commencer par une brève explicitation de deux expériences d'observation visuelle, réalisées par l'un et l'autre dans deux contextes différents de recherche. Nous avons retenu une première

\footnotetext{
4 Bouillet, A., 2001. Rudiments problématiques, À la recherche d'une éducation esthétique : rudiments, affinités, enjeux, Les Cahiers du Cerfee, 17.
}

expérience du géographe dans le Nordeste brésilien, une seconde de l'agronome dans le massif des Vosges du Sud.

\section{La clôture dans le Nordeste brésilien}

La clôture est peu à peu devenue aux yeux du géographe un marqueur des stratégies des éleveurs au regard du foncier et de la conduite du troupeau. Elle organise l'espace géographique. Le constat s'est forgé dans le cadre d'un projet de recherche-action mené dans la région semi-aride du Nordeste brésilien et visant à tester un ensemble de méthodes et d'instruments pour le développement de l'agriculture familiale ${ }^{5}$. Les premiers diagnostics réalisés dans le district de Massaroca (État de Bahia) conduisent à proposer la diffusion de techniques d'intensification fourragère pour résoudre le problème du déficit en saison sèche. L'analyse des pratiques montre que, de manière inattendue, la plupart des éleveurs utilisent les crédits obtenus pour clôturer de nouvelles terres et s'approprier ce qui était jusqu'alors en usage collectif. Le projet révèle combien l'enclosure devient le support de stratégies complexes d'appropriation privative et de mise en valeur de l'espace et des ressources pastorales. L'importance croissante des surfaces clôturées et la pression de pâturage modifient la conduite du troupeau. Le lâcher des ruminants sur parcours, sans gardiennage, est de plus en plus limité à la saison des pluies. L'alimentation des troupeaux évolue via la mobilisation raisonnée dans le temps de deux types d'espace complémentaires : l'espace clôturé, de propriété et d'usage individuels, cultivé ou non; l'espace ouvert à tous, constitué des parcours collectifs, des réserves foncières communautaires de droit coutumier destinées à l'installation des jeunes et des terres individuelles non clôturées. Les éleveurs exploitent le plus longtemps possible dans l'année, et grâce à un grand nombre d'animaux, la ressource fourragère des parcours en accès libre. Au sein de l'espace clôturé, les lots sont faits et défaits en fonction de l'état physiologique, corporel et éventuellement pathologique des animaux, du niveau de l'offre fourragère résiduelle des parcours et du pari fait sur la date des pluies suivantes.

\section{Les surfaces en herbe dans le massif vosgien}

Il s'agit de l'observation des paysages agricoles, à 30 ans d'intervalle, dans les années 1974-1976 et en 2005. En 1975, la question traitée par la recherche est celle de la déprise agricole dans le massif, posée par les professionnels agricoles et plus largement par la société vosgienne.

\footnotetext{
${ }^{5}$ Caron, P., 1998. Espaces, élevage et dynamique du changement: analyse, niveaux d'organisation et action. Le cas du Nordeste semiaride $d u$ Brésil. Thèse de doctorat en géographie et pratiques du développement, Université Paris 10, Nanterre.
} 
L'ouverture du paysage dépendrait de l'utilisation des surfaces en herbe, donc des exploitations et des pratiques d'élevage. On privilégie dans un premier temps l'observation visuelle pour appréhender la diversité des situations. Deux échelles d'observation sont retenues : celle de petits territoires et celle de la parcelle. L'analyse du paysage révèle des portions de territoire d'apparence relativement égale, «les terroirs». Ils sont ouverts, en déprise, ou déjà fermés par l'extension de la forêt et de la friche. L'observation des parcelles montre différentes pratiques d'utilisation de l'herbe, du "peigné vosgien », qui manifeste une utilisation attentive et soignée des prairies, aux friches herbacées dans les «essarts " communaux. Cette observation renvoie à des enquêtes en exploitations. Celles-ci diffèrent selon leur taille, leur avenir, leur système de pratiques, leurs résultats économiques et leur localisation dans le territoire. Cette analyse participe à un diagnostic plus général sur le développement de l'agriculture en relation avec les autres activités du massif. En 2005, le retour est moins approfondi ${ }^{6}$. Il reprend les niveaux d'observation du terroir, de la parcelle, et l'analyse de quelques exploitations pour saisir l'évolution des pratiques. Il révèle que le paysage des «Hauts » a peu changé bien que le "peigné vosgien » ait disparu. Les nouvelles pratiques mises en œuvre dans les surfaces en herbe dénotent moins de soins dans leur application. Dans les vallées, sauf en quelques endroits, le bâti et la forêt ont fermé le paysage. En 30 ans, le paysage est devenu un facteur de développement économique largement pris en compte par les collectivités locales, mais aussi par les agriculteurs dans leur projet d'exploitation. De nombreux signes visuels en témoignent.

\section{Une mise en correspondance des pratiques d'observation}

Chaque chercheur porte sur le paysage un regard particulier qu'il s'est construit progressivement. Ce regard singulier est lié à son questionnement, lui-même influencé par sa formation, sa personnalité, son histoire, le statut de l'espace et du paysage dans sa discipline. Pour le géographe, l'espace est objet central d'investigation, et ici d'observation, de manière à rendre compte de son organisation et de sa dynamique. Pour l'agronome, l'espace est un facteur parmi d'autres qui révèle la diversité des pratiques et permet de rendre intelligibles leur logique, leur évolution et leurs conséquences.

Par la mise en correspondance des pratiques d'observation et en nous appuyant sur les deux expériences brièvement présentées, nous avons cherché à expliciter quelques repères partagés. Nous en avons distingué sept. Ils ne représentent pas une succession d'étapes dans une

\footnotetext{
${ }^{6}$ Groupe de recherches INRA-ENESAD, à paraître. Pays, paysans, paysages, 30 ans après. Document.
}

démarche d'observation, mais plutôt des points de passage auxquels l'observateur est confronté dans sa pratique de l'analyse visuelle. Au préalable, il nous a semblé nécessaire de rappeler quelques propriétés du paysage indépendantes du voyant.

Le paysage observé est la matérialisation d'un espace discontinu et ce qui en est vu correspond à une partie seulement du territoire. Il est délimité par le choix, conscient ou non, de circonscrire le champ visuel, voire le champ d'observation. Il est composé d'une succession de plans, chacun à une échelle spatiale liée à la distance à l'observateur. Les composantes du paysage évoluent selon des pas de temps très divers. Des objets sont fugaces, comme les formes d'un troupeau au pâturage, d'autres plus durables, comme la texture et la couleur d'une surface en herbe ; d'autres, enfin, ont une relative pérennité, comme l'aménagement en terrasses de cultures en pente.

\section{Un questionnement}

Le questionnement, qui fonde et motive l'investigation, oriente et organise l'observation. Il s'agit du cadre général d'une problématique qui conditionne la démarche de travail. Dans ce cadre, des interrogations plus précises émergent et évoluent au cours de l'observation.

Dans l'expérience vosgienne, la question générale posée en 1975 est celle de la déprise agricole dans le massif. L'agronome choisit d'orienter sa recherche sur la diversité et la dynamique des pratiques d'utilisation des surfaces en herbe par l'élevage. Ce choix va orienter la démarche d'observation du paysage.

$\mathrm{Au}$ Brésil, le géographe cherche à accompagner les transformations économiques et sociales par une compréhension de l'organisation de l'espace. La clôture apparaît progressivement comme une clef de compréhension pertinente, non seulement des changements techniques, mais aussi des rapports sociaux.

\section{Un schéma pour l'observation}

Il ressort de la confrontation des deux itinéraires d'observation un schéma en trois pôles (Fig. 1). Le premier est celui des objets matériels dont les formes et les couleurs sont deux attributs visuels. Il peut être utile de distinguer différentes familles d'objets dans le paysage et de savoir les dénommer, en référence à différentes échelles d'espace et de temps. En s'inspirant de l'analyse que fait $R$. Barthes des objets décrits dans les planches de $l^{\prime} E_{n c y c l o p e ́ d i e}{ }^{7}$, où il discerne trois catégories : $1^{\prime}$ objet " qui est », l'objet «qui est fait», l'objet «qui fait», on peut différencier les « objets techniques » mobilisés par l'activité humaine pour réaliser des opérations techniques (le

\footnotetext{
7 Barthes, R., 1953. Les planches de l'Encyclopédie, in Le Degré zéro de l'écriture, Paris, Le Seuil, 89.
} 


\section{Objets (formes-couleurs)}

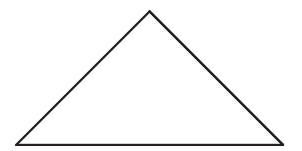

Structure des objets Organisation

Relations entre les objets Fonctionnement et processus Systèmes

Fig. 1. Les trois pôles de l'observation visuelle.

tracteur, l'outil...), «l'objet façonné » qui résulte de ces opérations (la haie, la parcelle) et «l'objet raisonné ${ }^{8}$, construit par l'observateur dans sa démarche d'analyse (ce qui se trouve à l'intérieur d'une surface clôturée, une unité d'égale apparence dans le paysage).

Un second pôle est celui des structures, c'est-à-dire de la répartition et de la configuration des objets dans l'espace. Ceux-ci sont mis en relation pour distinguer des processus, des fonctionnements, des dynamiques et des systèmes : c'est le troisième pôle.

Dans le cadre de ce schéma général, les démarches d'observation se construisent, fondées sur la question à traiter, sur les référents théoriques et sur l'expérience et les compétences de chacun. Certains objets (formescouleurs) et certaines structures s'avèrent des indicateurs visuels des fonctionnements et des processus. L'élaboration d'un schéma d'observation et la définition d'indicateurs sont à réviser en cours d'analyse et face à de nouvelles situations paysagères.

L'agronome privilégie deux échelles spatiales dans un diagnostic visuel. La première permet l'analyse de la parcelle d'usage agricole et de sa bordure (le champ d'observation est d'environ 10 ha). À cette échelle parcellaire, l'observation débute par un va-et-vient entre les objets (formes-couleurs) : la haie, une balle ronde de foin, l'abreuvoir... et la structure : dimension et forme de la parcelle, structure spatiale des objets... Les objets et les structures révèlent les façons concrètes dont les éleveurs mettent en œuvre les techniques, c'est-à-dire les pratiques : la fenaison, le mode de pâturage, la gestion de la fumure, la maîtrise de l'eau, l'entretien des bordures... autant d'éléments qui participent à un système technique. La seconde échelle (le champ d'observation est de 500 à 1000 ha) révèle la diversité des pratiques et des portions de territoire au sein desquelles il existe une relative uniformité des pratiques. Ces portions de territoire peuvent être cartographiées.

Au Brésil, un schéma d'observation est élaboré empiriquement et progressivement. L'analyse des structures agraires et la conduite d'enquêtes, de suivis

\footnotetext{
8 Deffontaines, J.-P., 2004. L'objet dans l'espace agricole. Le regard d'un géoagronome, Natures Sciences Sociétés, 12, 3, 299304.
}

d'exploitations et d'expérimentations fourragères confèrent un statut particulier à la clôture. Cette dernière devient un objet d'observation privilégié : sa pose intentionnelle traduit un acte stratégique; elle est facilement observable et la permanence de l'objet en fait un artéfact pertinent pour rendre compte d'évolutions stratégiques sur un pas de temps de plusieurs années. Ainsi, plusieurs critères sont choisis pour repérer et pour interpréter la diversité des actes associés à la clôture. Il s'agit de la localisation, du matériel utilisé (bois ou métal, nombre de fils) et de la technique de confection (tressage horizontal de piquets rapidement réalisé mais ne durant que deux saisons; piquets plantés dans le sol, plus exigeants en main d'œuvre mais plus solides ; écartement entre piquets dans le cas de fil de fer), de l'âge et de l'entretien. Ces critères traduisent des choix de gestion des ressources et d'affectation du territoire et rendent ainsi compte des priorités de l'éleveur. La compréhension de ces choix peut amener à élaborer de nouveaux indicateurs visuels : le type d'animaux contenus dans la parcelle clôturée, par exemple.

\section{Du détail au global, du proche au lointain et inversement}

L'observation est un va-et-vient. Ce qui s'avère essentiel est de distinguer différentes échelles d'espace, significatives pour le point de vue développé et au regard de la question posée, et de chercher à intégrer ce qui ressort de l'observation conduite à chacune de ces échelles. À chaque échelle correspondent des familles d'objets et des structures qui révèlent des fonctionnements particuliers.

Le va-et-vient entre les échelles contribue à l'intelligibilité des processus en cause. Il suppose une mobilité du regard du chercheur. " C'est le cheminement même du regard qui est productif ${ }^{9}$. »

Le terroir vosgien de la Colline présente, en 2005, une dominante d'herbe, quelques parcelles en déprise dans le haut des versants en contrebas de la forêt, un habitat dispersé. L'observation à l'échelle de la parcelle révèle des zones humides à proximité d'anciens bâtiments de ferme; les nombreux petits canaux non entretenus laissent se répandre l'eau librement. Le retour à l'échelle du terroir indique de fréquentes situations humides dans le versant. Les systèmes d'élevage d'aujourd'hui n'assurent plus la maîtrise de l'excès d'eau sur les versants où la fauche et le pâturage sont associés. Ceci se traduit par une dégradation progressive de la ressource fourragère.

Au Brésil, en élargissant le champ d'observation ou en se déplaçant, en voiture par exemple, on voit apparaître la dimension des parcelles encloses, les différences

\footnotetext{
${ }^{9}$ Marié, M., 2001. De la formation du regard dans les sciences humaines. Conférence du 18 janvier, séminaire "Ruralités contemporaines », EHESS, Paris.
} 
de la végétation de part et d'autre de la clôture (cultures et assolements, parcours, jachère), la localisation des animaux (quand ils sont visibles). Ces critères renseignent sur les aménagements dans le territoire.

\section{L'observation du temps}

Divers temps sont mêlés dans le paysage, le temps des objets et des traces, « le temps rond » des activités humaines et de la végétation, «le temps long» des processus géologiques ${ }^{10}$. Chaque point de vue disciplinaire retient des pas de temps estimés pertinents au vu de ses théories et de ses méthodes. Le géographe s'intéresse aux rythmes des changements des structures, l'agronome, aux transformations des façons de produire.

\section{Le temps de l'observation}

Prendre du temps! Le temps qu'il faut pour laisser les objets (formes-couleurs) devenir reconnaissables, pour mémoriser les paysages qu'évoque la vision; "c'est cet aller et retour entre le terrain et le voyage [...] qui donne la capacité d'étonnement ${ }^{11}$ ", le temps pour échanger avec les hommes qui habitent et transforment le paysage par leur activité.

Après un temps plus ou moins long, peut se produire un changement, parfois lié à une fréquence d'observation. Du sens apparaît, des liens s'établissent, une nouvelle question émerge, une suggestion de changement d'échelle d'espace ou de temps se manifeste, voire une certaine logique des structures dans le territoire, et même une certaine évidence.

Dans le cas du Brésil, l'intelligibilité n'aurait pu se construire sans une répétition fréquente de l'observation, sans un temps long, celui de l'observation, venant compléter dans la durée l'accompagnement par le chercheur des processus de changement technique et social.

\section{Les contrastes}

Un découpage de l'espace paysage, fondé sur les contrastes visuels, est un résultat de l'observation pour comprendre. Chaque observateur met en avant son propre découpage et propose une interprétation. Les unités spatiales, résultant de ce découpage, sont l'une des catégories d'objets dont la prise en compte contribue à

\footnotetext{
${ }^{10}$ Cf. Pierret, P., Deffontaines, J.-P., Landais, É., 2000. Le temps long et le temps rond des paysages agricoles, in Barrué-Pastor, M., Bertrand, G., Les Temps de l'environnement, Toulouse, Presses universitaires du Mirail, 335-342.

11 Marié, M., loc. cit.
}

la recherche d'une signification fonctionnelle. Les unités spatiales sont soit cartographiées (ce qui suppose une démarche complémentaire à l'observation du paysage pour informer la totalité du territoire), soit inscrites sur un fond de paysage (croquis ou photo). Sur cette base, peut s'organiser une lecture plus globale des processus passés ou en cours. Ce sont donc deux types de résultats auxquels conduit l'observation : des produits concrets et tangibles que l'on peut donner à voir (croquis, cartes, etc.) et des connaissances sur les processus à l'œuvre.

La validation des observations peut passer par des méthodes non fondées sur le visuel (analyse statistique, enquête auprès d'acteurs locaux, bibliographie...). Mais elle peut également reposer sur la constitution d'indicateurs observables ou sur un «croisement des regards».

Dans certaines localités du Nordeste brésilien, l'espace est marqué par une appropriation individuelle presque totale des terres de parcours. La clôture n'y a pas les mêmes fonctions qu'à Massaroca. Il est, par exemple, des lieux où ce sont les éleveurs qui clôturent pour contrôler les divagations de leurs animaux et non plus les agriculteurs pour prévenir les dégâts. Le sens du filtre est alors inversé et la technique de clôture différente. Cela entraîne des changements techniques conséquents, pouvant aller jusqu'à la disparition de l'élevage caprin qui nécessite la réalisation de clôtures à 9 rangées de fil de fer barbelé. Ailleurs encore, la clôture a essentiellement pour fonction de marquer la propriété foncière. Elle peut alors, à l'image des planteurs de manioc réduits à cultiver les interstices entre route et latifundia d'élevage extensif, «offrir » des signes tangibles d'exclusion sociale. La validation du schéma d'observation se fait donc localement. L' « universalité » des clefs de compréhension reste toute relative et celles qui sont valables à Massaroca ne facilitent pas nécessairement l'interprétation des faits ailleurs. La transposition du schéma d'observation permet tout à la fois de mieux cerner le domaine de validité du schéma lui-même et de le faire évoluer.

\section{Croisement des regards}

Le croisement des regards est ici une pratique particulière de l'observation qui consiste à comparer ce qui est vu par différents observateurs devant un paysage. L'expérience nous amène à souligner l'intérêt de cette pratique. Pour comprendre les dynamiques écologiques et sociales d'agricultures locales, le croisement des regards entre sciences sociales et biotechniques peut se faire dans une phase initiale de l'analyse. L'observation du paysage est alors utile pour établir des liens entre les disciplines engagées. Le paysage apparaît comme un facilitateur de dialogue entre points de vue scientifiques différents, eux-mêmes portés par des théories, des méthodes et des pratiques particulières. De même, en cours 
d'analyse, la pratique de mise en relation de ce qui est vu par chacun permet d'élargir le champ explicatif proposé par chaque discipline. Par ailleurs, en permettant une approche par le visible de la complexité des terrains, le croisement des regards facilite une valorisation de l'observation en termes d'action pour l'aménagement et le développement.

Ce croisement exige de procéder par étapes. Une fois l'espace d'observation borné et définie l'échelle de travail par les différents observateurs, s'opère la confrontation des objets, décrits par leurs attributs, et des découpages. Le choix d'échelles semblables est nécessaire, car il est peu utile de mettre en parallèle la partition d'un paysage en quelques unités spatiales par un observateur, avec une partition en nombreuses unités par un autre. La mise en commun des interprétations élaborées par chacun, en argumentant par retour sur les objets et les hypothèses mises en avant, représente la pièce maîtresse de ce croisement.

Quels sont les changements de l'activité agricole observés, en 2005, dans les terroirs vosgiens du Haut et à quoi les attribuer? L'agronome observe de nouveaux objets (formes-couleurs) : la race vosgienne traditionnelle des troupeaux de vaches laitières, qui avait disparu, remplace la race Holstein, pourtant plus productive ; les communaux ne sont plus en friche mais entretenus par les troupeaux de vosgiennes plus rustiques; la limite avec la forêt de conifères qui coiffe les versants est plus nette. L'agronome distingue des ensembles de parcelles, contiguës ou non, dont le couvert présente une texture et une couleur semblables. Ces ensembles dénotent des pratiques voisines. Leur disposition dans l'espace suggère une segmentation du paysage.

Sur ce même paysage, le géographe relève la présence de nombreuses habitations traditionnelles dispersées, récemment remises en état, et ne présentant plus aucun des objets de proximité qui caractérisent le siège de l'exploitation agricole. De nouveaux acteurs interviennent dans le paysage, des résidents ou des touristes. La multiplication de ces nouveaux usagers du territoire n'est-elle pas à l'origine de nouvelles exigences paysagères pour le cadre de vie (recherche d'identité des lieux, de paysages lisibles et soignés, de produits locaux de qualité), qui se traduisent par de nouvelles stratégies de production, d'accueil, de type de produits et de filières? $C^{\prime}$ est par enquête que des réponses sont apportées à ces questions.

Il se confirme deux évolutions majeures : le développement du tourisme et de la résidence; l'apparition de nouvelles attentes de la société vosgienne en termes de qualité du territoire et des produits. Cette évolution se traduit par de nouveaux choix techniques et de nouvelles stratégies territoriales, notamment au niveau des exploitations agricoles et des collectivités locales.

\section{Réflexions sur l'observation pour comprendre}

Le regard du chercheur participe d'une démarche d'observation et, plus largement, $d^{\prime}$ investigation et de quête d'intelligibilité des faits inscrits dans le paysage. Nous nous situons ainsi dans le cadre d'une observation visuelle «sur le terrain». Le paysage est vu sans l'intermédiaire de techniques de vision, telles que l'image photographique, télévisuelle ou de synthèse.

L'observation de terrain évoquée donne une consistance au réel et s'oppose à « l'abstraction inéluctable du visue $^{12} »$. Il s'agit d'un regard attentif pour comprendre. Le paysage peut être vu comme un ensemble complexe d'objets, plus ou moins durables, agencés dans l'espace. Parmi l'infinité des objets visibles, chaque chercheur retient ou construit ceux qui lui paraissent significatifs au regard de son questionnement. Certains procèdent par " concrétion d'objets", d'autres par inventaire et classification. Ces objets s'inscrivent dans des systèmes écologiques et sociaux dont ils révèlent des aspects structurels, fonctionnels ou dynamiques.

Les modalités de l'observation visuelle soulèvent diverses questions d'ordre épistémologique et méthodologique. Un premier point concerne les relations qu'entretiennent le rationnel et le sensible. Le sujet regardant est un tout où interagissent connaissance et sensibilité ; en observant, j'apprends et je ressens tout à la fois. L'expérience montre que la science peut éveiller et alimenter la sensibilité. Pour A. de Humboldt, «la rationalité scientifique est peu à peu devenue capable [...] de contribuer elle-même à stimuler et enrichir la contemplation de la nature $^{13}$ ». La connaissance modifie le sentiment de plaisir ressenti face au paysage. Mais on peut aussi se référer à Merleau-Ponty, pour qui «la science manipule les choses et renonce à les habiter ${ }^{14}$ ». Dans certains contextes, la connaissance peut étouffer l'émotion.

Qu'en est-il du lien inverse du sensible vers la science? Pour A. de Humboldt, «les œuvres directement issues de l'imagination poétique deviennent, dans la mesure où elles portent le sentiment spontanément éprouvé de l'unité de l'univers, de véritables outils de diffusion du message scientifique lui-même et de propagation du goût de la science ${ }^{15} »$. Chez cet auteur, tout est sans doute dans cette conception de l'unité de l'univers. Sa posture face au cosmos où poètes et scientifiques

\footnotetext{
12 Baudrillard, J., Nouvel, J., 2000. Les Objets singuliers : architecture et philosophie, Paris, Calmann-Lévy.

13 Briffaud, S., 2006. Le temps du paysage. Alexandre de Humboldt et la géohistoire du sentiment de la nature, in Blais, H., Laboulais, I., Géographies plurielles : les sciences géographiques au moment de l'émergence des sciences humaines (1750-1850), Paris, L'Harmattan, 275-301 (cit. p. 281).

14 Merleau-Ponty, M., op. cit.

15 Briffaud, S., 2006, ibidem.
} 
parlent ensemble et s'écoutent pour saisir ce « tout » est à la fois une utopie et peut-être une direction. Pour ce qui est du paysage, nous constatons que la relation sensible qui s'établit au cours de l'observation n'est pas sans influence sur le mode d'acquisition de la connaissance. Au cours de l'analyse d'un paysage, il est fréquent de chercher à dissimuler et à ignorer la dimension sensible par référence à des normes de scientificité. En effet, le paysage comme outil de connaissance est aussi objet d'impressions de la part de l'observateur. Il évoque, émeut, symbolise, suggère des jugements de valeur. La première impression devant un paysage a une qualité que l'on ne retrouve pas une fois engagée la démarche d'analyse. Cette impression immédiate et éphémère peut avoir une valeur pour la connaissance et participer à l'intelligibilité. Il nous semble important de chercher à la valoriser, à la garder en mémoire, estimant qu'elle peut avoir une valeur heuristique et prendre part à la démarche rationnelle. Parfois, l'observation débouche sur une connivence avec le paysage, une sorte d'adhésion ; la distance aux objets observés s'estompe. On est dans le paysage. La question se pose de savoir si cette connivence représente un frein à la poursuite de l'analyse visuelle pour comprendre. La dimension visuelle correspondrait-elle à « la synthèse passive » dont parle Husserl ${ }^{16}$, qui précède l'analyse et se distingue de la "synthèse active », ou encore au concept de «préréflexif » de Merleau-Ponty?

La dimension sensible renvoie ainsi à la notion de « présence » : présence d'un paysage au même titre que celle de certains tableaux, mais aussi présence de soi dans le paysage, «cette impression non pas de voir mais d'être vu par ces objets ${ }^{17}$ ». Dans l'observation du paysage, il y a une implication du corps. Le face-à-face avec lui n'estil pas aussi un corps à corps? Si le visuel domine, tous les sens sont mobilisés, plus ou moins volontairement. L'observateur est immobile ou en mouvement, il est seul ou avec d'autres.

Le «questionnement » et le «schéma d'observation » renforcent l'idée que l'observateur n'est pas un spectateur qui assiste, mais que le regardant se conforme à une sorte de convention initiale ; il s'inscrit dans un système plus ou moins formalisé de limites, voire de règles ${ }^{18}$. Le système doit cependant rester ouvert et essentiellement évolutif pour ne pas réduire la capacité à saisir l'inattendu.

Ces considérations épistémologiques interrogent en retour les éléments de méthode élaborés dans la confrontation de nos pratiques. Notre réflexion se fonde sur la notion de croisement des regards. Nous l'avons vu, cette

\footnotetext{
${ }^{16}$ Husserl, E., 1998. De la synthèse passive : logique transcendantale et constitutions originaires, Grenoble, J. Million.

17 Valéry, P., 2000. Poésies, in Poésie perdue : les poèmes en prose des Cahiers, Paris, Gallimard, 103.

18 Cf. Crary, J., 1990. L'Art de l'observateur : vision et modernité au XIX $X^{e}$ siècle, Nîmes, J. Chambon.
}

notion présente deux significations dans ce propos. Le croisement des regards désigne, d'une part, la confrontation des pratiques d'observation d'un géographe et d'un agronome. Cette confrontation a permis de distinguer quelques traits communs à ces pratiques. Ils concernent le questionnement qui oriente l'analyse visuelle, un schéma pour l'observation, le va-et-vient entre le proche et le lointain, l'approche du temps, la recherche des contrastes, le croisement des regards et la dimension sensible dans l'observation pour comprendre. Ces rubriques ne sont pas des étapes dans un itinéraire d'observation, mais plutôt des repères pour l'observateur, des points de passage ponctuant des itinéraires et des séquences cognitifs à chaque fois renouvelés en fonction du propos et de l'intention.

Le croisement des regards est, d'autre part, une analyse comparée de ce que révèle un paysage à des observateurs différents. On est souvent seul devant un paysage et on peut tenter soi-même de mobiliser les connaissances acquises dans différents domaines et chercher à les croiser. Lorsque l'on observe à plusieurs, on est d'abord surpris par la vision de l'autre et par une complexité inattendue contenue dans le paysage. Cette multiplicité des visions est une découverte pour qui est attentif au regard de l'autre. Une prise de conscience première pour le croisement des regards est que le paysage contient tous les visibles de tous les voyants.

Dans le propos qui précède, sont mis en relation les points de vue de deux chercheurs sur le paysage, mais l'expérience montre que le croisement peut s'opérer dans des finalités différentes avec divers acteurs. Par exemple, dans la perspective de l'élaboration d'un projet de territoire dans un lieu, élus, habitants, membres d'associations, visiteurs, experts peuvent échanger leurs regards sur le paysage, chacun privilégiant certains aspects qui sont mis en débat. Le paysage est médiateur entre les perceptions et entre les diverses stratégies territoriales.

Dans la perspective d'une intelligibilité des pratiques territoriales des agriculteurs et des éleveurs, nous pensons important de mieux connaître les modes d'observation visuelle développés tout au long des activités de production, notamment dans la conduite de leur système de culture et d'élevage. Dans ce but et en prolongement de ce qui est développé dans ce propos, nous avons engagé un travail de recherche avec un berger et une anthropologue.

Parmi les questions de méthode, se posent également celle de la place de la mesure et des outils (cartes, photos, dessins...) et celle du rôle des autres sens que la vue dans les pratiques d'observation.

Pour ce qui est de la transmission du savoir issu de l'observation, et celle du comment observer, nous retenons quelques réflexions et interrogations. Il apparaît difficile de se défaire de l'idée «qu'observer cela va de 
soi $^{19}$ ». Comment dire ce que l'on voit, sachant que nous partageons ce que nous voyons par ce que nous disons voir? Comment initier au désir d'observer, de s'étonner, de s'interroger sur ce qui semble aller de soi ? «Comment échapper à la passivité de la vision ${ }^{20} »$ ? Une question apparaît ainsi à l'issue de l'échange sur nos pratiques d'observation, celle de l'intérêt et de la possibilité d'élaborer une théorie de l'observation visuelle. Le partage d'expériences auquel nous avons procédé y contribue peut-être.

\footnotetext{
19 Bouillet, A., 2001. loc. cit.

20 Bachelard, G., 1948. La Terre et les rêveries du repos, Paris, José Corti.
} 